\section{A 20-year experience of electron microscopy in the diagnosis of primary ciliary dyskinesia}

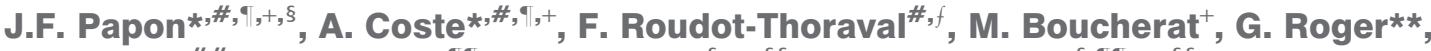 \\ A. Tamalet ${ }^{\# \#}$, A.M. Vojtek ${ }^{\tau \uparrow}$, S. Amselem ${ }^{\S,++, \S}$ and E. Escudier ${ }^{\S, \uparrow \uparrow,++, \S \S}$
}

ABSTRACT: Transmission electron microscopy (TEM) analysis of ciliary ultrastructure is classically used for the diagnosis of primary ciliary dyskinesia (PCD). We report our extensive experience of TEM analysis in a large series of patients in order to evaluate its feasibility and results.

TEM analysis performed in 1,149 patients with suspected PCD was retrospectively reviewed. Biopsies $(1,450)$ were obtained from nasal $(44 \%)$ or bronchial $(56 \%)$ mucosa in children $(66.5 \%)$ and adults $(33.5 \%)$.

TEM analysis was feasible in $71.4 \%$ of patients and showed a main defect suggestive of PCD in 29.9\%. TEM was more feasible in adults than in children, regardless of the biopsy site. Main defects suggestive of PCD were found in $76.9 \%$ of patients with sinopulmonary symptoms and in only $0.4 \%$ of patients with isolated upper and $0.4 \%$ with isolated lower respiratory tract infections. The defect pattern was similar in children and adults, involving dynein arms $(81.2 \%)$ or central complex (CC) (18.8\%). Situs inversus was never observed in PCD patients with CC defect. Kartagener syndrome with normal ciliary ultrastructure was not an exceptional condition $(10.2 \%$ of PCD).

In conclusion, TEM analysis is feasible in most patients and is particularly useful for PCD diagnosis in cases of sinopulmonary syndrome of unknown origin.

KEYWORDS: Airways, cilia, dynein, Kartagener syndrome, situs inversus, ultrastructure

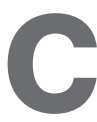

ilia, evolutionarily conserved structures, are classified according to their cytoskeleton core called axoneme: primary cilia with sensory function, and motile cilia ensuring fluid transport. Defects in primary cilia have been associated with a growing number of rare genetic diseases (polycystic kidney disease, Bardet-Biedl syndrome and retinitis pigmentosa), whereas motile cilia are involved in the most prominent ciliopathy called primary ciliary dyskinesia (PCD) [1].

PCD is a congenital disorder with an estimated prevalence of 1:15-30,000 live births [2] and is due to impaired mucociliary transport resulting from a lack of ciliary motion leading to chronic respiratory infections. The clinical features of PCD, usually beginning in early childhood, are characterised by bronchiectasis and chronic sinusitis, sometimes associated with situs inversus and male sterility [3]. The axoneme of motile cilia is composed of nine peripheral doublet microtubules with attached inner and outer dynein arms (IDA and ODA, respectively) and radial spokes, surrounding a central complex (CC) consisting of two central microtubules surrounded by the central sheath. PCD is a heterogeneous group of genetic disorders usually transmitted as autosomal recessive traits with various ciliary ultrastructural defects [3]. The absence of pathognomonic clinical and laboratory signs makes PCD difficult to diagnose. However, it is of prime importance to recognise this disease in order to start appropriate therapy of respiratory tract infections and minimise lung damage. In this context, the finding by Afzelius [4] that most respiratory cilia of patients with PCD carry ultrastructural defects has opened up new ways to manage this disease, especially by providing the first objective test of diagnostic value.

Transmission electron microscopy (TEM) analysis of cilia is still a relevant technology now frequently combined with new innovative investigations for the diagnosis of PCD. However, TEM analysis is an arduous and expensive
AFFILIATIONS

*INSERM, U955,

\#Universite Paris 12, Faculte de Medecine, UMR S841,

'AP-HP, Groupe Hospitalier HenriMondor - Albert Chenevier, Service d'ORL et de Chirurgie Cervicofaciale,

+Hopital Intercommunal, Service d'ORL et de Chirurgie Cervicofaciale,

${ }^{f}$ AP-HP, Groupe Hospitalier HenriMondor - Albert Chenevier, Service de Sante Publique

"Hopital Intercommunal, Service d'Anatomo-pathologie (Laboratoire de Microscopie Electronique), Creteil,

${ }^{\S}$ INSERM, U933 Paris,

${ }^{\star *}$ AP-HP, Hopital Armand-

Trousseau, Service d'ORL et de

Chirurgie Cervico-faciale Pediatrique,

\#\# AP-HP, Hopital Armand-

Trousseau, Service de Pneumologie

Pediatrique,

+Universite Paris 6, Faculte de

Medecine, and

${ }^{\S \S} \mathrm{AP}-\mathrm{HP}, \mathrm{Hopital}$ Armand-Trousseau

Service de Genetique et

d'embryologie Medicales, Paris,

France.

CORRESPONDENCE

J.F. Papon,

Consultation ORL, Hopital H.

Mondor, 51, avenue du Marechal de Lattre de Tassigny, 94010 Creteil

Cedex, France

E-mail: jean-francois.papon@

hmn.aphp.fr

Received:

March 222009

Accepted after revision:

Sept 292009

First published online:

Oct 192009

European Respiratory Journal

Print ISSN 0903-1936

Online ISSN 1399-3003 
technique performed in highly selected patients, requiring airway biopsies that are processed by multiple technical steps. More importantly, TEM can be defective for analysis of ciliary ultrastructure even after optimal processing. Few studies based on large series of patients (i.e. $>50$ patients) with PCD have been reported [5-7] and none of them have focused on the efficacy of TEM analysis of cilia. It therefore seemed important to report our lengthy experience of TEM analysis of cilia based on a large series of patients. The feasibility and results of TEM analysis of cilia performed in our institution from 1985 to 2006 were retrospectively analysed in the present study.

\section{PATIENTS AND METHODS \\ Patients}

Physicians experienced in respiratory endoscopy from several hospitals consecutively sent airway biopsies to our laboratory and these samples were consecutively examined for TEM analysis of cilia. Airway biopsies were performed in patients with respiratory tract infections suggestive of PCD in two main clinical situations: 1) highly suggestive presentation of PCD (i.e. situs inversus with airway infections); and 2) recurrent airway infections (i.e. rhinosinusitis, bronchitis, bronchiectasis) after exclusion of all known pathological conditions (such as cystic fibrosis or immunodeficiency). The physicians performed biopsies after at least 30 days absence of exacerbation of respiratory tract infection and, if necessary, at the end of an antibiotic course.

\section{Ciliary ultrastructure}

Biopsies were obtained from either bronchial (main bronchus) or nasal (inferior turbinate) mucosa of children ( $<18$ yrs of age) or adults. Children with suspected PCD were essentially referred to paediatric respiratory physicians who mainly performed bronchial biopsies, whereas adults with suspected PCD were essentially referred to ENT physicians who performed nasal biopsies. Patients or their parents were informed by their physicians of the exact nature and goal of all investigations performed and gave their informed consent.

Airway biopsies were immersed in $2.5 \%$ glutaraldehyde and processed as usual for ultrastructural analysis [8]. Ultrathin sections were examined at a final magnification of $\times 60,000$ without knowledge of the clinical data. In each specimen, analysis of at least 50 transverse ciliary sections of different cells was required to study the internal axonemal structure according to a quantitative method [9]. Ciliary ultrastructure results were expressed as a percentage of abnormal cilia among the total number of cilia analysed. As previously reported, up to $10 \%$ of cilia in control specimens can exhibit ultrastructural defects $[10,11]$. For this study, ciliary abnormalities were defined as the presence of $>20 \%$ of ciliary defects. For each ciliary study, axonemal abnormalities were quantified and expressed as a percentage of each ultrastructural defect over the total number of abnormal cilia to define the main ultrastructural defect. The main ultrastructural defect can concern ODA (total absence or short ODA isolated or associated with absence of IDA), IDA (absence of IDA isolated or associated with radial spoke defect) or CC (central microtubules absent or single) abnormalities. Since 2002, for questionable IDA in micrographs obtained by TEM, computerised analysis of cilia was systematically performed to improve IDA visualisation, as previously reported [12]. Ciliary orientation was systematically evaluated by comparing the position of the central pairs of adjoining cilia as previously described $[11,13]$. Disorientation was defined as an angle $>25^{\circ}$.

\section{Statistical analysis}

Results are expressed as numbers and percentages for categorical data, and as median with interquartile range (IQR) and range for quantitative data with a non-normal distribution. Main results were expressed with their 95\% confidence interval (CI). Comparisons of categorical data were performed with Chi-squared test or Mantel-Haenszel statistics when adjusted on a third variable and quantitative data were compared by Kruskal-Wallis nonparametric test. The relationship between site of infection and main defect was tested by Chi-square test. A p-value $<0.05$ was considered significant.

\section{RESULTS}

\section{TEM feasibility}

1,149 patients (corresponding to 1,450 biopsies) were examined for ciliary ultrastructure. TEM was unfeasible (see below) in 329 patients $(28.6 \%)$ and feasible in 820 patients $(71.4 \%)$, constituting the study population. Among the 329 patients in whom TEM was unfeasible (corresponding to 464 (32\%) out of 1,450 biopsies), 207, 111 and 11 patients were biopsied once, twice or three or more times, respectively. The first biopsy did not provide samples of sufficient quality for TEM analysis in 459 patients (39.9\%). Among these patients, 252 (54.9\%) received a second biopsy providing samples of sufficient quality in 98 patients (38.9\%). Finally, among the 154 patients with unfeasible TEM in the second sample, 43 (27.9\%) received a third biopsy providing samples of sufficient quality for TEM in $32(74.4 \%)$ patients.

We found that feasibility of TEM analysis did not vary with year (table 1). Among the 1,450 airway biopsies, 636 (44\%) were obtained from nasal mucosa and 814 (56\%) from bronchial mucosa. TEM was significantly more feasible on nasal $(458 / 636,72 \%)$ than on bronchial $(528 / 814,64.8 \%)$ biopsies $(\mathrm{p}<0.03)$. Among these 1,450 biopsies, $965(66.5 \%)$ were performed in children and 485 (33.5\%) were performed in adults. TEM was significantly more feasible in biopsies from adults $(424 / 485,87.4 \%)$ than in biopsies from children (562/ $965,58.2 \%)(p<0.001)$. Within the children's group, the TEM was significantly more feasible in the 10-17-yr-old range than in the $0-9$-yr-old range $(\mathrm{p}<0.05)$ (table 1$)$. Nasal biopsies were performed more frequently in adults $(322 / 485,66.4 \%)$ and bronchial biopsies were performed more frequently in children (651/965, 67.5\%). The difference of TEM feasibility between nasal and bronchial biopsies was no longer observed when adjusted for the patient's age $(p=0.33)$, while the difference between adults and children persisted when adjusted for biopsy site $(\mathrm{p}<0.001)$.

The reasons for TEM unfeasibility in nasal biopsies were squamous metaplasia (50\%), denuded basement membrane without ciliated cells (25\%), rarefaction of cilia (19\%) and cell alterations $(6 \%)$. The reasons for TEM unfeasibility in bronchial biopsies were rarefaction of cilia $(42 \%)$, denuded basement membrane without ciliated cells $(27 \%)$, very small number of epithelial cells in the biopsy (12\%), squamous metaplasia (10\%) and cell alterations (9\%). 


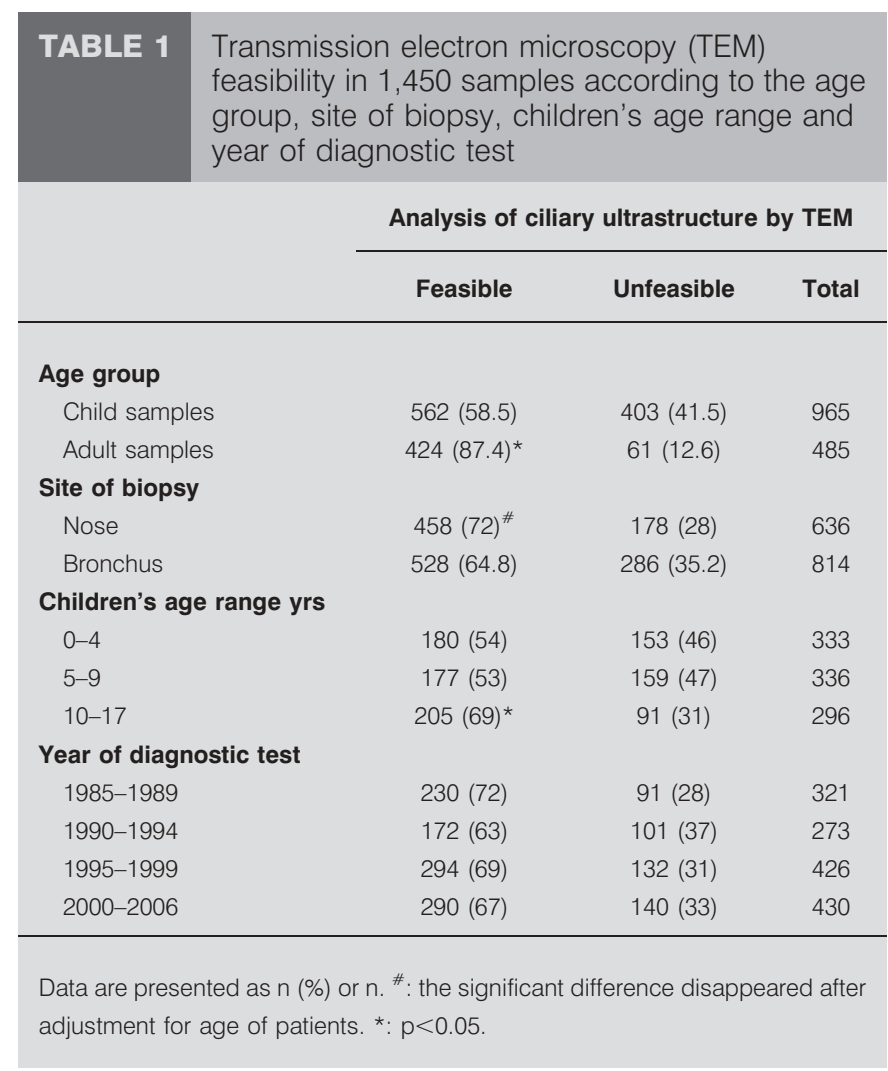

\section{Analysis of ciliary ultrastructure}

Ciliary ultrastructure was analysable in 820 patients $(71.4 \%)$ constituting the study population composed of 467 children (mean age: $8.1 \pm 4.8$ yrs, range: $0.2-17.8$ ) and 353 adults (mean age: $41.3 \pm 14.4$ yrs, range: $18-70.4)$.

TEM analysis showed normal ciliary ultrastructure (group I) in 533 patients $(65 \%)$ (fig. 1a), heterogeneous ciliary abnormalities without a main ultrastructural defect (group II) in 15 patients $(1.8 \%)$, abnormal cilia with a main ultrastructural defect (group III) in 245 patients $(29.9 \%$ ) and questionable ciliary ultrastructure (group IV) in 27 patients (3.3\%) even after computerised analysis of cilia. In fact, computer-assisted analysis of micrographs, performed for 95 patients, allowed us to reach a conclusion for 68 patients (15 and 53 patients belonging to groups I and III, respectively). The median, IQR and range of percentage of abnormal cilia in each group are given in table 2 .

The children/adults ratio was significantly different between groups $(\mathrm{p}<0.001)$ corresponding to a higher proportion of children in group III (table 2). Although present in each group, situs inversus was significantly more frequent in group III $(31.8 \%)$ than in the other groups $(\mathrm{p}<0.0001)$ (table 2$)$.

Although only limited clinical information was provided by most of the physicians who performed the biopsies, the topography of upper and/or lower airway infections could be studied in the various groups. Interestingly, $90 \%$ of patients in group I and II suffered from chronic infections exclusively involving either upper or lower airways (table 2). In contrast, $99.2 \%$ of patients in group III suffered from a combination of upper and lower airway infections, i.e. sinopulmonary syndrome that was significantly more frequent in this group than in the other groups $(\mathrm{p}<0.001)$ (table 2$)$. A main defect suggestive of PCD was found in $243(76.9 \%)$ of the 316 patients with sinopulmonary syndrome and in two $(0.4 \%)$ of the 504 patients without sinopulmonary syndrome. These two patients were a child with severe asthma without upper airway infection, and an adult with situs inversus and nasal polyposis without lower airway infection. In group I, 28 patients with normal ciliary ultrastructure had the clinical criteria to diagnose Kartagener syndrome (i.e. association of sinusitis, bronchiectasis and situs inversus) and we considered them as Kartagener syndrome with normal ciliary ultrastructure.

In group I, the percentage of abnormal cilia was very low, as previously reported in controls [10]. In group II, the heterogeneous ciliary abnormalities mostly concerned the peripheral microtubules. In group III (table 3), the main ultrastructural defect concerned ODA in $64.9 \%$ of patients, IDA alone in $16.3 \%$ of patients and CC in $18.8 \%$ of patients. Situs inversus was significantly more frequent in patients with ODA than in patients with IDA defects and was never observed in patients with CC abnormalities ( $\mathrm{p}<0.001)$. The median, IQR and range of abnormal cilia in patients with ODA, IDA and CC defects are given in table 3 . ODA abnormalities $(n=159)$ were either isolated (total absence or short ODA) in 81 patients (fig. 1b) or associated with absence of IDA in 56 patients (fig. 1c); in the remaining 22 patients, the ODA defect was associated with questionable IDA (fig. 1d). Absence of IDA alone $(n=40)$ was either isolated (nine patients) (fig. 1e) or associated with radial spoke defect (31 patients) (fig. 1f). Interestingly, in group III, only $142 / 245$ patients (58\%) exhibited $100 \%$ of abnormal cilia and the abnormalities always involved the dynein arms and never the CC (table 3). The main ultrastructural defect in children from group III concerned ODA, IDA and CC for $61.2 \%, 18.2 \%$ and $20.6 \%$ patients, respectively. The main ultrastructural defect in adults from group III concerned ODA, IDA and CC for $72.5 \%, 12.5 \%$ and $15 \%$ patients, respectively.

In group IV, with questionable ultrastructure, even after computerised analysis of cilia, most difficulties concerned analysis of IDA (24/27 patients, $88.9 \%$ ) (fig. $1 \mathrm{~g}$ ), whereas analysis of ODA was questionable in only three out of 27 patients (11.1\%) and CC analysis was never a problem (fig. 1h).

The results of ciliary orientation remained within the normal range in the four groups and were not related to ultrastructural abnormalities (data not shown).

\section{DISCUSSION}

We report our 20-yr experience of TEM analysis based on a historical series of $>1,000$ patients with suspected PCD, indicating the limitations of the technique that was not feasible in nearly one-third of patients, but also demonstrating that TEM remains a useful tool for the diagnosis of PCD, providing a precise ultrastructural phenotype in most cases. Our study highlights that PCD with CC defects and Kartagener syndrome with normal ciliary ultrastructure are not exceptional conditions. TEM analysis is particularly useful in the case of sinopulmonary syndrome of unknown origin, especially for identification of PCD with CC defects, a condition that is never associated with situs inversus. 

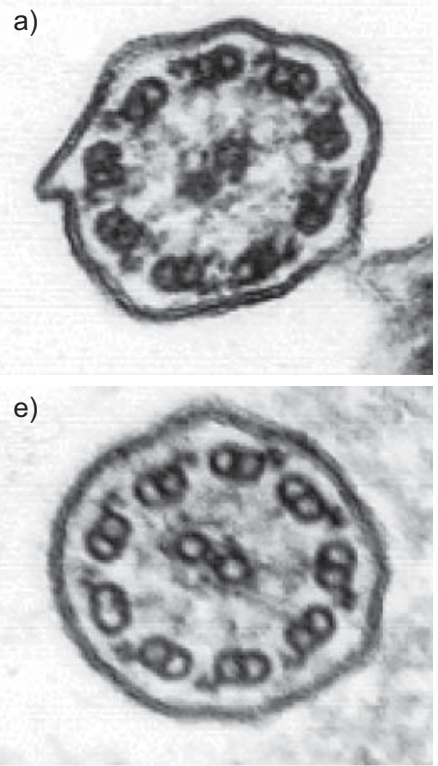

b)

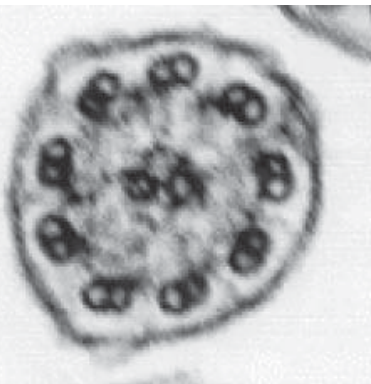

f)

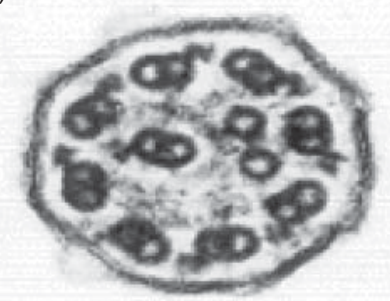

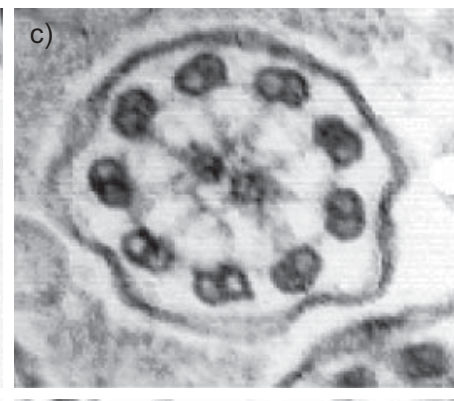
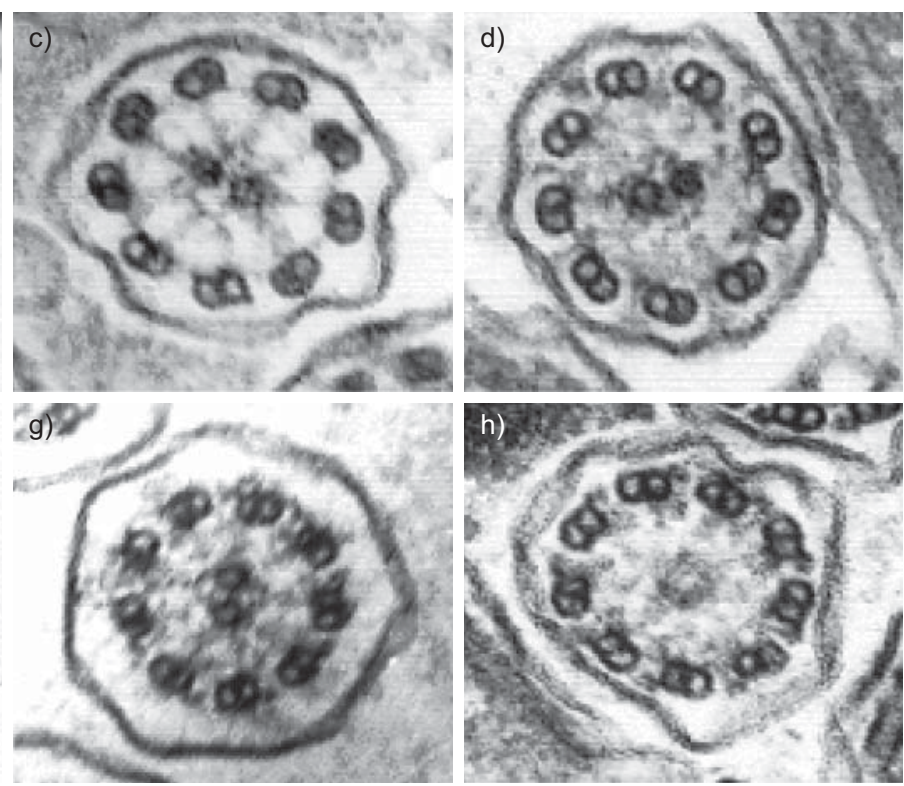

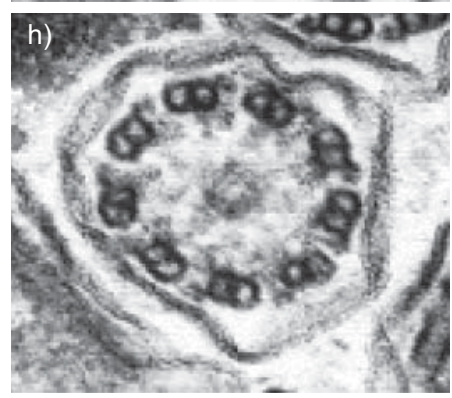

FIGURE 1. Representative images of the ultrastructure of respiratory cilia. a) Cross-section of normal cilia showing the "9+2" microtubule doublet configuration with presence of dynein arms. b) Absence of outer dynein arms. c) Absence of both dynein arms. d) Absence of outer dynein arms and questionable inner dynein arms. e) Absence of inner dynein arms. f) Absence of inner dynein arms and axonemal disorganisation. g) Questionable inner dynein arms. h) Absence of central microtubules. Magnification $\times 60,000$.

In our series of 1,450 airway biopsies, the TEM failure rate was comparable with that of previous studies [14, 15]. Epithelial metaplasia and denuded basement membrane were the main reasons for failure in nasal and bronchial biopsies, respectively. The site of biopsy for ciliary studies has rarely been studied in the literature, except for one paediatric study that also showed a high frequency of metaplasia in nasal biopsies [16]. Analysis showed a similar TEM feasibility between nasal and bronchial biopsies, regardless of the age. Interestingly, TEM analysis was significantly more feasible in biopsies from adults than in

TABLE 2 Characteristics of the groups as defined by ciliary ultrastructure in the 820 patients with feasible transmission electron microscopy analysis

\begin{tabular}{|c|c|c|c|c|c|}
\hline & \multicolumn{4}{|c|}{ Groups } & \multirow[t]{2}{*}{$p$-value } \\
\hline & I & II & III & IV & \\
\hline Patients & $533(65)$ & $15(1.8)$ & $245(29)$ & $27(3.3)$ & \\
\hline Median & 5 & 28.5 & $100^{\#}$ & ND & $<0.0001$ \\
\hline IQR & $2-10$ & $25-34$ & $51-100$ & ND & \\
\hline Range & 0-20 & $21-42$ & $20.5-100$ & ND & \\
\hline Sex ratio $F / M$ & 0.68 & 0.36 & 0.78 & 0.8 & NS \\
\hline Children/adults ratio & 1.09 & 0.87 & $2.06^{\#}$ & 1.7 & $<0.001$ \\
\hline Situs inversus & $28(5.2)$ & $1(6.7)$ & $78(31.8)^{\#}$ & $2(7.4)$ & $<0.0001$ \\
\hline
\end{tabular}

Data are presented as $n(\%)$, unless otherwise indicated. IQR: interquartile range; F/M: female/male; ND: not determined; NS: nonsignificant. p-values are for global comparison between the four groups; if $p<0.05$, two-by-two comparisons were performed. ${ }^{*}$ : indicates that only this group was significantly different from the other groups; ": indicates that the two groups were not different from each other but were significantly different from the other two groups. 


\begin{tabular}{|c|c|c|c|c|c|}
\hline \multirow[t]{3}{*}{ TABLE 3} & \multicolumn{5}{|c|}{$\begin{array}{l}\text { Phenotypic features according to the main ciliary } \\
\text { defects as defined by transmission electron } \\
\text { microscopy (group III, } n=245 \text { ) }\end{array}$} \\
\hline & & \multicolumn{3}{|c|}{ Main ultrastructural defect } & \multirow[t]{2}{*}{$\mathrm{p}$-value } \\
\hline & & ODA & IDA & CC & \\
\hline \multirow{2}{*}{\multicolumn{2}{|c|}{$\begin{array}{l}\text { Patients } \mathbf{n} \\
\text { Associated defects } \\
\text { IDA }\end{array}$}} & 159 & 40 & 46 & \\
\hline & & & & & \\
\hline \multicolumn{2}{|c|}{ Normal } & 81 & & & \\
\hline \multicolumn{2}{|c|}{ Defect } & 56 & & & \\
\hline \multicolumn{2}{|c|}{ Questionable } & 22 & & & \\
\hline \multicolumn{6}{|c|}{ Radial spoke } \\
\hline \multicolumn{2}{|c|}{ Normal } & & 9 & & \\
\hline \multicolumn{2}{|c|}{ Defect } & & 31 & & \\
\hline \multicolumn{6}{|c|}{ Abnormal cilia \% } \\
\hline \multicolumn{2}{|c|}{ Median } & $100^{\#}$ & $100^{\#}$ & 37 & $<0.0001$ \\
\hline \multicolumn{2}{|l|}{ IQR } & $78-100$ & $100-100$ & $26.7-60.2$ & \\
\hline \multicolumn{2}{|l|}{ Range } & $21-100$ & $30-100$ & $20.5-85$ & \\
\hline \multicolumn{2}{|c|}{ Sex ratio F/M } & 0.91 & 0.66 & 0.65 & NS \\
\hline \multicolumn{2}{|c|}{ Children/adults ratio } & 2.74 & 3 & 2.83 & NS \\
\hline \multicolumn{2}{|c|}{ Situs inversus } & $65(40.9)^{\bullet}$ & $13(32.5)$ & $0(0)^{\circ}$ & $<0.001$ \\
\hline \multicolumn{2}{|c|}{$\begin{array}{l}\text { Patients with } 100 \% \\
\text { abnormal cilia }\end{array}$} & $110(69.2)$ & $32(80)$ & $0(0)^{+}$ & $<0.001$ \\
\hline
\end{tabular}

Data are presented as $n(\%)$, unless otherwise indicated. ODA: outer dynein arm; IDA: inner dynein arm; CC: central complex; IQR: interquartile range; F/M: female/male; Ns: nonsignificant. p-values are for global comparison between the four groups; if $p<0.05$, two-by-two comparisons were performed. ${ }^{*}$ : indicates that the two groups were not different from each other but were significantly different from the other two groups; $"$ : indicates that the two groups were different from each other and were also significantly different from the other two groups; ${ }^{+}$: indicates that only this group was significantly different from the other groups.

biopsies from children, probably due to the technical challenge of taking biopsies of the narrow airways of children, accounting for the small size of biopsies. In fact, when TEM analysis was unfeasible after the first sampling, the repetition of biopsies seemed to be helpful and the interval between biopsies appeared to be less important than the absence of exacerbation of respiratory tract infections.

In the 820 patients in whom TEM analysis was feasible, ciliary ultrastructure was normal in more than one-half of patients, which raises the issue of accurate patient selection for TEM analysis of cilia. Situs inversus associated with airway infections is the most suggestive condition justifying systematic TEM analysis of cilia. However, situs inversus is not constant in PCD [5-7, 17] and ciliary studies are often proposed in the presence of upper and/or lower chronic airway infections of unknown origin. The strong relationship between the presence of a sinopulmonary syndrome and the detection of a main defect suggests that this syndrome could be a good clinical criterion to improve patient selection for TEM analysis of cilia. However, all other known pathological conditions must be excluded before performing TEM analysis, which may be unfeasible in about one-third of these patients.
In line with the literature, the main ciliary defect (group III) concerned dynein arms in $>80 \%$ of cases and more frequently concerned ODA than IDA in adults and in children $[6,7]$. Our results, based on the study of a very large series of patients, describe a spectrum of ultrastructural defects in PCD similar to those reported in other studies (i.e. isolated ODA defects: 24 $43 \%$; ODA associated with IDA defects: $24-45 \%$; isolated IDA defects: $14-29 \%$; CC defects: 4-18\%) [6, 7, 18]. In most patients with a main ciliary defect, all cilia were abnormal, as expected for a congenital disease. However, some normal cilia can persist, rarely associated with dynein arm defects but always in the case of CC defects [9, 19]. Although ultrastructural defects are usually not detected in more than half of the cilia, CC defects are considered to be congenital [3, 9, 20]. The constitutional nature of this specific defect is demonstrated by the existence of sibling forms of PCD with CC defects [20] and similar abnormalities described in Chlamydomonas mutants, a cellular model for PCD [21-24]. In patients with CC defects, the presence of about one-half of normal cilia can be explained by various hypotheses: 1) instability of central microtubules [25], 2) short length of central microtubules only present in the basal part of the cilia [22], or 3) quantitative synthesis deficiency providing central microtubule structures for only some cilia.

Patients with heterogeneous abnormalities of cilia (group II) were uncommon; these abnormalities concerned the number of peripheral microtubules, and are considered to be acquired ciliary defects related to recurrent airway damage [20, 26]. TEM analysis of cilia in cultured respiratory epithelial cells has been proposed to eliminate such heterogeneous abnormalities of cilia [18].

In very few patients (group IV), TEM analysis was feasible but ultrastructure was questionable. As previously reported, IDA is the most frequent questionable axonemal structure [19, 27] because of its low contrast on TEM. The use of computerassisted analysis of micrographs greatly improves dynein arm visualisation in doubtful cases after classical TEM [12].

It is noteworthy that situs inversus was observed in $<50 \%$ of patients with a main ciliary defect. This result, in agreement with previous data [6], is lower than the classically reported proportion of situs inversus [5, 7, 17]. This discrepancy could be due to patient selection mainly based on the presence of chronic airway infections of unknown origin, but not necessarily associated with situs inversus. Finally, our study showed that the proportion of situs inversus varies with the type of ultrastructural defect, is more frequent in the case of abnormal ODA than IDA, and is never associated with CC defects, as already mentioned $[6,20]$. The absence of situs inversus in the case of CC defects could be explained by the fact that visceral lateralisation is initiated by nodal cilia that normally do not contain a central pair of microtubules. It is therefore not surprising that a molecular defect involving the central microtubules does not modify visceral lateralisation.

Finally, Kartagener syndrome with normal cilia was not an exceptional condition, found in 28 of 109 patients with situs inversus. Similarly, based on random lateralisation, it can be speculated that our series should contain the same proportion of "true" patients with PCD but with normal cilia and without situs inversus. Strikingly, 25 patients from group I with normal 
cilia (and without situs inversus) exhibited sinopulmonary syndrome. Overall, an estimated $18 \% \quad((28+25=53) /(28+25+$ $245=298)$ ) of patients with PCD may exhibit normal cilia. Due to the absence of ciliary ultrastructural defects in these patients, the diagnosis of PCD requires additional diagnostic tools.

Due to the long observation period, new diagnostic tools have become available during that time that enhance patient selection for TEM analysis and/or improve diagnostic procedures. Considering the limitations of TEM analysis stressed in this study (i.e. unfeasible in nearly $30 \%$ of the patients and Kartagener syndrome with normal ciliary ultrastructure), the diagnosis of PCD now relies on the association of TEM analysis and these tools $[2,28]$. In addition to the evaluation of ciliary beat frequency, which frequently remains the first investigation for the diagnosis of PCD [29], high-resolution, digital highspeed videomicroscopy has been developed to characterise abnormal beat patterns specific for axonemal defects $[6,7,30]$. Nasal nitric oxide is dramatically reduced in most patients with confirmed PCD [7, 31]. Immunostaining methods using antibodies directed against the main axonemal components are also developed to facilitate identification of structural abnormalities of cilia [32]. Lastly, identification of genes involved in the pathogenesis of PCD would be an ideal tool. This approach remains challenging because PCD is genetically heterogeneous with numerous candidate genes that are sometimes very large. Two main genes, DNAI1 and DNAH5 have been identified to date $[33,34]$ for PCD with isolated ODA defects and the other identified genes (i.e. RPGR, TXNDC3, DNAH1, DNAI2, KTU, RSPH9 and RSPH4A) concern a few PCD families [35-40]. The identification of the ciliary ultrastructural defect by TEM analysis could also be helpful for genetic analysis.

In conclusion, TEM is often feasible and very useful in defining the ultrastructural phenotype, particularly in the case of sinopulmonary syndrome of unknown origin. In patients with isolated upper or lower respiratory tract infections, TEM should be performed only if there is a very high level of suspicion. TEM is especially helpful for the diagnosis of PCD when situs inversus is absent as we constantly found in PCD with CC defects. Our results also highlight that the spectrum of ultrastructural defects is similar in children and adults and that Kartagener syndrome with normal ciliary ultrastructure is not an exceptional condition, representing a technical challenge for the diagnosis of PCD. Our study also stresses the limitations of solely using ultrastructural analysis in PCD diagnosis, which is currently based on convergent elements derived from clinical phenotype, TEM results and additional diagnostic tools, and is essential to start early appropriate therapy designed to prevent lung damage.

\section{SUPPORT STATEMENT}

This work was supported by grants from the Legs Poix from the Chancellerie des Universites, the Assistance Publique-Hopitaux de Paris (PHRC AOM06053, P060245) and the Agence Nationale pour la Recherche (ANR-05-MRAR-022-01).

\section{STATEMENT OF INTEREST}

None declared.

\section{ACKNOWLEDGEMENTS}

The authors are grateful to the patients and their families who participated in this study. We would also like to thank B. Escudier for careful review and useful advice for writing this manuscript.

\section{REFERENCES}

1 Badano JL, Mitsuma M, Beales PL, et al. The ciliopathies: an emerging class of human genetic disorders. Annu Rev Genomics Hum Genet 2006; 7: 125-148.

2 Bush A, Chodhari R, Collins N, et al. Primary ciliary dyskinesia: current state of the art. Arch Dis Child 2007; 92: 1136-1140.

3 Afzelius BA. The immotile-cilia syndrome: a microtubule-associated defect. CRC Crit Rev Biochem 1985; 19: 63-87.

4 Afzelius BA. A human syndrome caused by immotile cilia. Science 1976; 193: 317-319.

5 Coren ME, Meeks M, Morrison I, et al. Primary ciliary dyskinesia: age at diagnosis and symptom history. Acta Paediatr 2002; 91: 667-669.

6 Chilvers MA, Rutman A, O'Callaghan C. Ciliary beat pattern is associated with specific ultrastructural defects in primary ciliary dyskinesia. J Allergy Clin Immunol 2003; 112: 518-524.

7 Noone PG, Leigh MW, Sannuti A, et al. Primary ciliary dyskinesia: diagnostic and phenotypic features. Am J Respir Crit Care Med 2004; 169: 459-467.

8 Verra F, Fleury-Feith J, Boucherat M, et al. Do nasal ciliary changes reflect bronchial changes? An ultrastructural study. Am Rev Respir Dis 1993; 147: 908-913.

9 Escalier D, Jouannet P, David G. Abnormalities of the ciliary axonemal complex in children: an ultrastructural cinetic study in a series of 34 cases. Biol Cell 1982; 44: 271-282.

10 Rossman C, Lee R, Forrest J, et al. Nasal ciliary ultrastructure and function in patients with primary ciliary dyskinesia compared with that in normal subjects and in subjects with various respiratory diseases. Am Rev Respir Dis 1984; 129: 161-167.

11 De Iongh RU, Rutland J. Ciliary defects in healthy subjects, bronchiectasis, and primary ciliary dyskinesia. Am J Respir Crit Care Med 1995; 151: 1559-1567.

12 Escudier E, Couprie M, Duriez B, et al. Computer-assisted analysis helps detect inner dynein arm abnormalities. Am J Respir Crit Care Med 2002; 166: 1257-1262.

13 Rautiainen M, Collan Y, Nuutinen J. A method for measuring the orientation ("beat direction") of respiratory cilia. Arch Otorhinolaryngol 1986; 243: 265-268.

14 Pizzi S, Cazzato S, Bernardi F, et al. Clinico-pathological evaluation of ciliary dyskinesia. Ultrastruc Pathol 2003; 27: 243-252.

15 Carda C, Armengot M, Escribano A, et al. Ultrastructural patterns of primary ciliary dyskinesia syndrome. Ultrastruc Pathol 2005; 29: 3-8.

16 MacCormick J, Robb I, Kovesi T, et al. Optimal biopsy techniques in the diagnosis of primary ciliary dyskinesia. J Otolaryngol 2002; 31: 13-17.

17 Kennedy M, Omran H, Leigh MW, et al. Congenital heart disease and other heterotaxic defects in a large cohort of patients with primary ciliary dyskinesia. Circulation 2007; 115: 2814-2821.

18 Jorissen M, Willems T, Van der Schueren B, et al. Ultrastructural expression of primary ciliary dyskinesia after ciliogenesis in culture. Acta Otorhinolaryngol Belg 2000; 54: 343-356.

19 Rutland J, Cox T, Dewar A, et al. Screening for ciliary dyskinesia a spectrum of defects of motility and structure. Eur J Respir Dis Suppl 1983; 127: 71-77.

20 Tamalet A, Clement A, Roudot-Thoraval F, et al. Abnormal central complex is a marker of severity in the presence of partial ciliary defect. Pediatrics 2001; 108: E86.

21 Eliasson R, Mossberg B, Cammer P, et al. The immotile-cilia syndrome. A congenital abnormality as an etiologic factor in chronic airway infections and male sterility. N Engl J Med 1977; 297: 1-6.

22 Afzelius BA, Eliasson R. Flagellar mutants in man: on the heterogeneity of the immotile-cilia syndrome. J Ultrastruct Res 1979; 69: 43-52.

23 Huang B, Luck D. Paralysed flagella mutants of Chlamydomonas reinhardtii defective fot axonemal doublet microtubule arms. J Biol Chem 1979; 254: 3091-3099. 
24 El Zein L, Omran H, Bouvagnet P. Lateralization defects and ciliary dyskinesia: lessons from algae. Trends Genet 2003; 19: 162-167.

25 Dentler WL. Isolation and charaxterization of ciliary membranes from Tetrahymena pyriformis. J Cell Biol 1980; 84: 364-380.

26 Torkkeli T, Nuutinen J, Rautiainen M. Clinical relevance of tubulus anomalies and compound cilia. Acta Otolaryngol Suppl 1997; 529: 140-143.

27 Chao J, Turner JA, Sturgess JM. Genetic heterogeneity of dyneindeficiency in cilia from patients with respiratory disease. Am Rev Respir Dis 1982; 126: 302-305.

28 Leigh MW, Zariwala MA, Knowles MR. Primary ciliary dyskinesia: improving the diagnostic approach. Curr Opin Pediatr 2009; 21 320-325.

29 Bush A, Cole P, Hariri M, et al. Primary ciliary dyskinesia: diagnosis and standards of care. Eur Respir J 1998; 12: 982-988.

30 Stannard W, Rutman A, Wallis C, et al. Central microtubular agenesis causing primary ciliary dyskinesia. Am J Resp Crit Care Med 2004; 169: 634-637.

31 Lundberg JO, Weitzberg E, Nordvall SL, et al. Primarily nasal origin of exhaled nitric oxide and absence in Kartagener's syndrome. Eur Respir J 1994; 7: 1501-1504.

32 Fliegauf M, Olbrich H, Horvath J, et al. Mislocalization of DNAH5 and DNAH9 in respiratory cells from patients with primary ciliary dyskinesia. Am J Respir Crit Care Med 2005; 171: 1343-1349.

33 Pennarun G, Escudier E, Chapelin C, et al. Loss-of-function mutations in a human gene related to Chlamydomonas reinhardtii dynein IC78 result in primary ciliary dyskinesia. Am J Hum Genet 1999; 65: 1508-1519.

34 Olbrich H, Haffner K, Kispert A, et al. Mutations in DNAH5 cause primary ciliary dyskinesia and randomization of left-right asymmetry. Nat Genet 2002; 30: 143-144.

35 Moore A, Escudier E, Roger G, et al. RPGR is mutated in patients with a complex $\mathrm{X}$ linked phenotype combining primary ciliary dyskinesia and retinitis pigmentosa. J Med Genet 2006; 43: 326-333.

36 Duriez B, Duquesnoy P, Escudier E, et al. A common variant in combination with a nonsense mutation in a member of the thioredoxin family causes primary ciliary dyskinesia. Proc Natl Acad Sci USA 2007; 104: 3336-3341.

37 Schwabe GC, Hoffmann K, Loges NT, et al. Primary ciliary dyskinesia associated with normal axoneme ultrastructure is caused by DNAH11 mutations. Hum Mutat 2008; 29: 289-298.

38 Loges NT, Olbrich H, Fenske L, et al. DNAI2 mutations cause primary ciliary dyskinesia with defects in the outer dynein arm. Am J Hum Genet 2008; 83: 547-558.

39 Omran $\mathrm{H}$, Kobayashi $\mathrm{D}$, Olbrich $\mathrm{H}$, et al. Ktu/PF13 is required for cytoplasmic pre-assembly of axonemal dyneins. Nature 2008; 456: 611-616.

40 Castleman VH, Romio L, Chodhari R, et al. Mutations in radial spoke head protein genes RSPH9 and RSPH4A cause primary ciliary dyskinesia with central-microtubular-pair abnormalities. Am J Hum Genet 2009; 84: 197-209. 